\title{
A Novel Mechanism for Low Bit-Rate Compression
}

\author{
K. Sripal Reddy ${ }^{1}$, S. Srinivas ${ }^{2}$ \\ ${ }^{I}$ (Department of DECS, Vardhaman College of Engineering, India) \\ ${ }^{2}$ (Department ofDECS, Vardhaman College of Engineering, India)
}

\begin{abstract}
Sparse sampling techniques have been around in digital photography. In this approach compression follows oversampling. In this paper a uniform down sampling is practically implemented. Moreover it supports adaptive sampling with low-pass and varying pre-filtering. The result of this can be compressed and then sent across to destination without making changes to current image. A decoder is built in MATLAB which decompresses image using the low-pass pre-filtering approach. The proposed approach is known as adaptive down sampling and up conversion which is far better than JPEG 2000. The prototype application is built to evaluate this fact. The proposed system exhibited low-bit rates with higher visual quality. The results revealed that the proposed method can be used in real world applications.
\end{abstract}

Index Terms-Image processing, up conversion, pre-filtering, and adaptive sampling.

Accepted Date: 26 June 2013

\section{Introduction}

Image processing has been around for many years. These domain applications are prevalent in many areas of application development. As the images are to be transferred over network or Internet, compression techniques came into existence. The conventional approach has been challenged by the researchers of later [1]. Compact image representation is investigated in this paper in spatial domain using sparse sampling. Most of the existing technologies use compact representation of images using interpolation. However, there is difficulty with high frequency contents. This challenge is overcome by the proposed image compression system which is known as collaborative adaptive down sampling and up conversion. It emphasizes an edge reconstruction which has got importance perceptually. In this mechanism we also avoid irregular down sampling. The proposed compression technique uses square pixel grid of image. The proposed technique can also be used with any existing compression techniques such as JPEG 2000 and DCT - based JPEG. Directional low-pass pre-filtering approach is used for uniform down sampling. The decoder in the proposed system actually decompresses the images which have low resolution. Afterwards it gets up converted into original image. Predictive image coding [2], [3] was used earlier for two - dimensional aggressive modeling. However, in the proposed system, the decoder plays an important role for predicting and also adapting. The predictor is used only at the decoder side in the proposed approach. Fig. 1 shows the same. DCT-based JPEG image compression technique is used in [4] with respect to critical bit rate. Down sampling technique was also proposed in that paper. However, it is not similar to proposed system as it is tailored and thus supports only codec pertaining to DCT-based JPEG. Later Gan et al. proposed pre and post filters in order to overcome artifacts which are revealed in case of DCT-based block codecs with given bit rates [5]. Thus the previous works are motivated by the fact that DCT-based codecs produce artifacts. In the proposed system the experiments revealed that the high bit rate applications exhibited less superiority while the low bit rate applications exhibited superior performance. With respect to PSNR and image visual quality, the proposed system outperforms the existing JPEG 2000 standard for image compression. The work done in [6] is similar to our work. However, it won't accept quincunx image as input for compression. Its encoder is also computationally expensive as it requires adaptive directional lifting. When compared with proposed one, the quincunx image processing has revealed that the proposed system

The rest of the paper is organized as follows. Section II provides insights into the proposed compression system which outperforms the JPEG 2000 standard. Section III provides results and analysis while the section $\mathrm{V}$ concludes this paper.

\section{Proposed Compression System}

The proposed compression system outline is presented in fig. 1. It has many components such as directional pre-filtering, uniform down sampling, third party encoder, third party decoder, auto-regressive modeling and up conversion. 


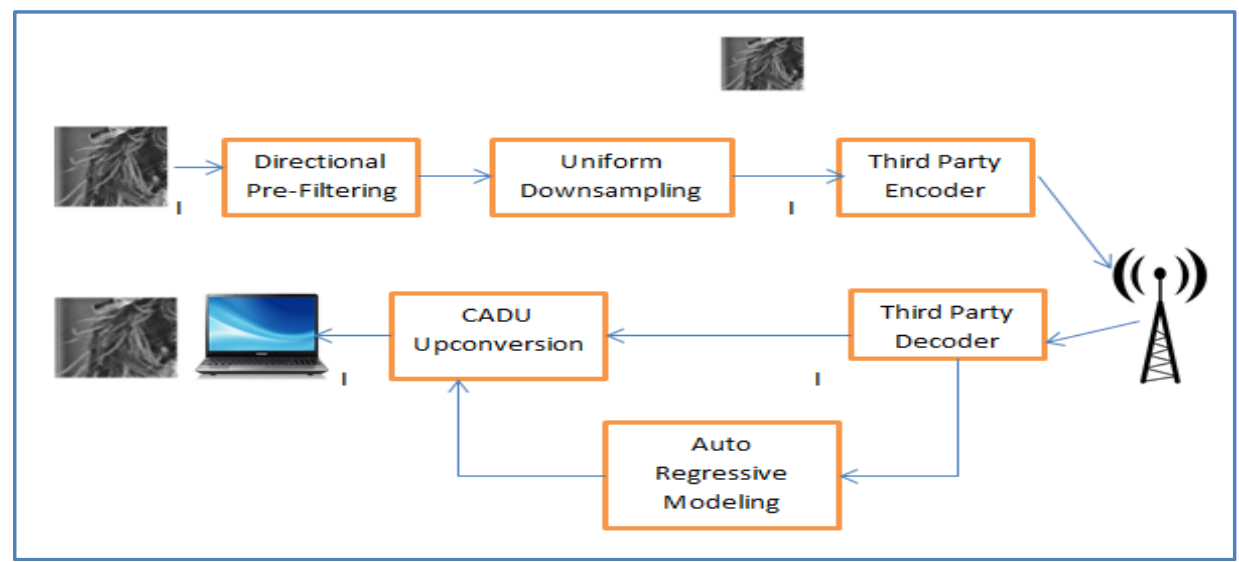

Fig. 1 -Proposed Image Compression Technique

As can be seen in fig. 1, the proposed compression technique is with encoding and decoding activities. It operates on down-sampled images. The results of this technique revealed best visual quality and PSNR. Thus the proposed technique is far better than that of JPEG 2000 though the latter is widely used codec [7]. The proposed compression technique also works with other third party compression techniques. The proposed system can extend any existing techniques to improve compression for low-bit rate performance. Visual communication in wireless networks in the real world is one of the applications of the proposed compression technique. When compared with it, the JPEG 2000 is inefficient as it consumes more bandwidth and can't exhibit any performance improvement for low-resolution devices.

The proposed compression technique reduces the complexity of encoder with any codec being used. This is very useful in reducing energy consumption. This technique is required in image processing operations being performed in wireless sensor networks. Many researches are relevant to our paper. They include [8] and [5]. Down sampling an image before it is subjected to JPEG compression and then up sampling is performed in [8]. The influence of bit rate in image compression techniques is presented by [4]. Interpolation and decimation algorithms were implemented in [9].

Directional low-pass filtering is achieved using the following equation.

$$
h_{\theta}(i, j)=m \operatorname{sinc}\left(\frac{i \cos \theta+j \sin \theta}{s_{i}}\right)
$$

The proposed compression technique is superior to JPEG 2000 compression technique. Its total mean-squares distortion is computed as follows.

$$
\left\{\begin{array}{l}
R(D)=\sum_{k=1}^{K} \max \left\{0, \frac{1}{2} \log _{2} \frac{\sigma_{k}^{2}}{\tau}\right\} \\
D(R)=\sum_{k=1}^{K} \min \left\{\tau, \sigma_{k}^{2}\right\}
\end{array}\right. \text {. }
$$

Rate distortion function satisfies the following condition and thus it outperforms the JPEG 2000 compression.

$$
D\left(r^{*}\right)>\int_{\pi / 2}^{\pi} \Phi(\omega) d \omega
$$

\section{Experimental Results And Evaluation}

The experiments are made using MATLAB in a PC with 2GB or RAM and Dual Core processor. The experiments are made using various bench mark images. The experiments are done using the proposed approach and compared with JPEG 2000. The results of the proposed compression method applied to Lena, Leaves and Bike images. The PSNR is computed and presented in table 1. 


\begin{tabular}{|l|l|l|l|}
\hline Image & Rate(bpp) & JPEG & $\begin{array}{l}\text { CADU- } \\
\text { JPG }\end{array}$ \\
\hline Lena & 0.10 & N/A & 27.69 \\
& 0.15 & 25.57 & 29.48 \\
& 0.20 & 28.77 & 30.72 \\
& 0.25 & 30.58 & 31.66 \\
& 0.30 & 31.81 & 32.48 \\
\hline Leaves & 0.10 & N/A & 23.83 \\
& 0.15 & N/A & 25.61 \\
& 0.20 & 24.14 & 26.78 \\
& 0.25 & 26.64 & 27.87 \\
& 0.30 & 27.86 & 28.73 \\
\hline Bike & 0.10 & N/A & 20.20 \\
& 0.15 & N/A & 21.25 \\
& 0.20 & 20.74 & 21.97 \\
& 0.25 & 21.84 & 22.53 \\
& 0.30 & 22.66 & 23.03 \\
& \multicolumn{3}{|l}{} \\
\hline
\end{tabular}

Table 1 - Results of JPEG 2000 and Proposed Compression Technique

As can be seen in table 1, it is evident that the proposed system exhibited superior features in terms of PSNR and image visual clarity. The results of the images which were taken when experimented with Lena, Leaves and Bike images are presented in fig. 2.

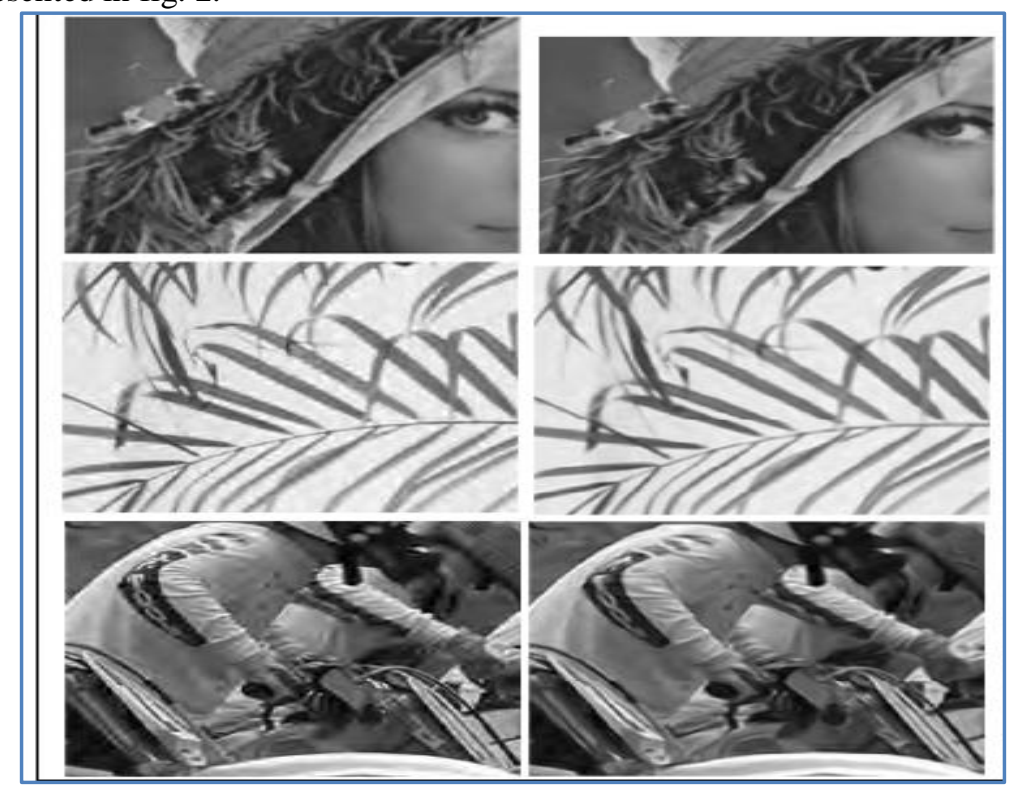

Fig. 2 - Difference in PSNR of Lena, Leaves and Bike

As can be seen in fig. 2, the left image is compressed with JPEG while the right image is compressed with proposed compression system. As can be seen in table 1, the results reveal that the proposed system outperforms the JPEG technique for image compression. Fig. 3, 4, and 6 present the difference is PSNR of Lena, Leaves and Bike images respectively for JPEG and proposed compression techniques. 


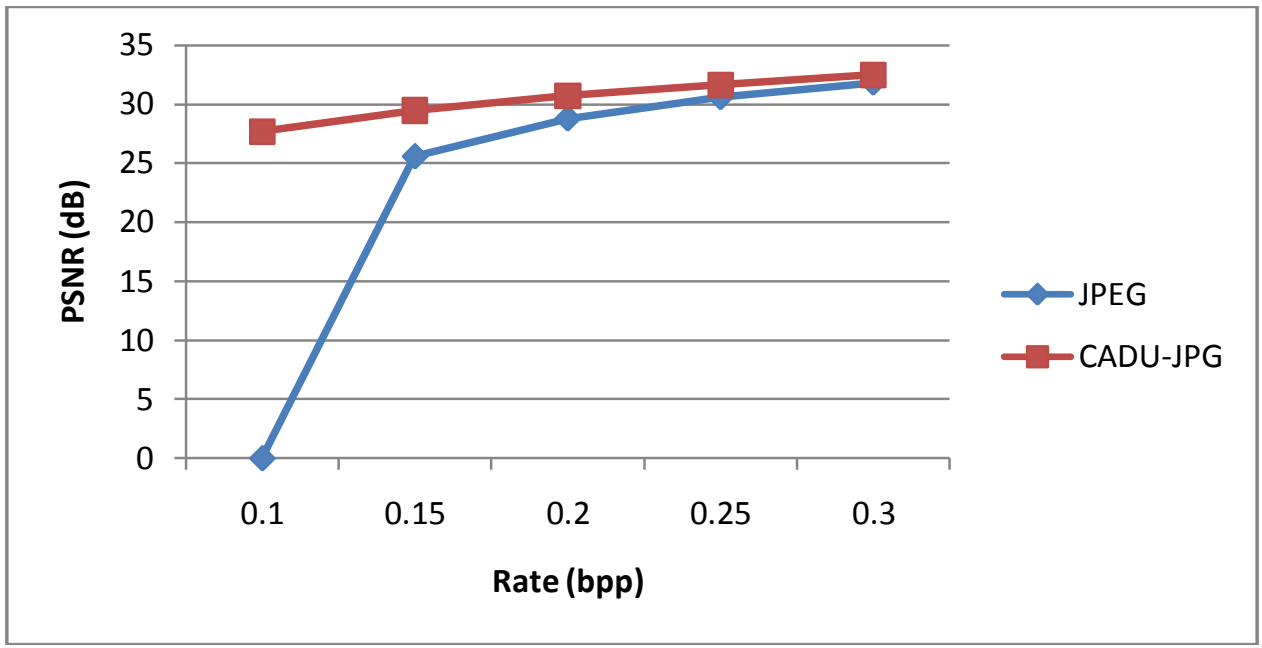

Fig. 3 -Comparison of Techniques With Respect to PSNR with Various Bit Rates(Lena)

As can be seen in fig 3, the horizontal axis represents bit rate while the vertical axis represents PSNR values. The results reveal that when bit rates are low the compression performance is higher. The proposed approach outperforms the JPEG compression method.

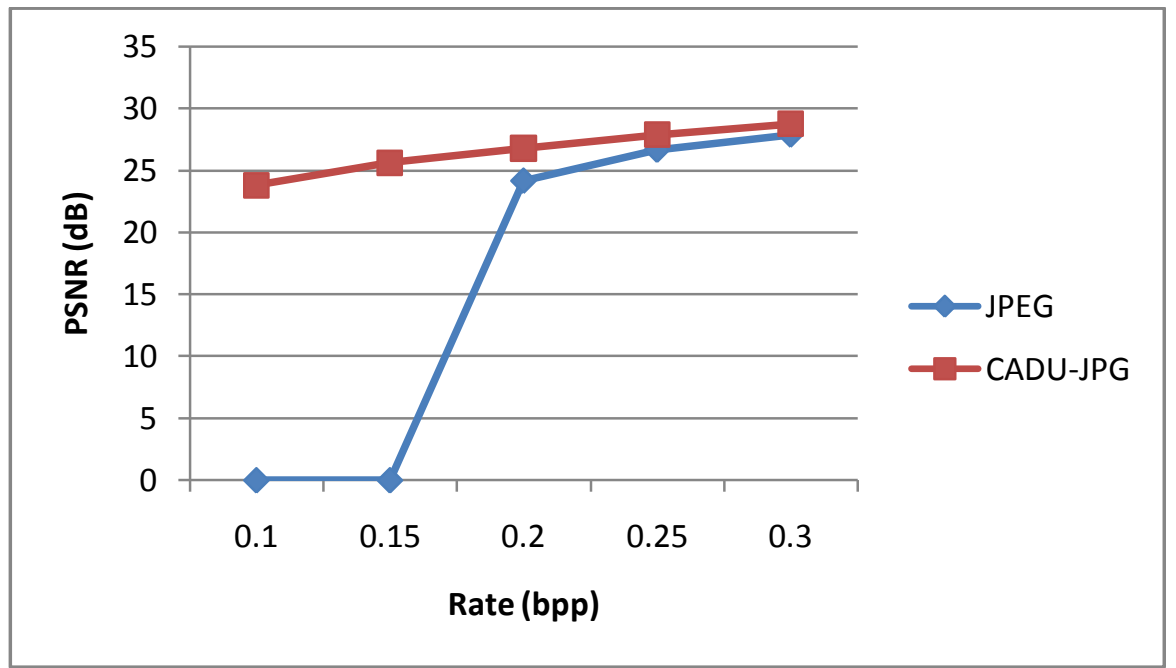

Fig. 4 - Comparison of Techniques With Respect to PSNR with Various Bit Rates (Leaves)

As can be seen in fig 4, the horizontal axis represents bit rate while the vertical axis represents PSNR values. The results reveal that when bit rates are low the compression performance is higher. The proposed approach outperforms the JPEG compression method.

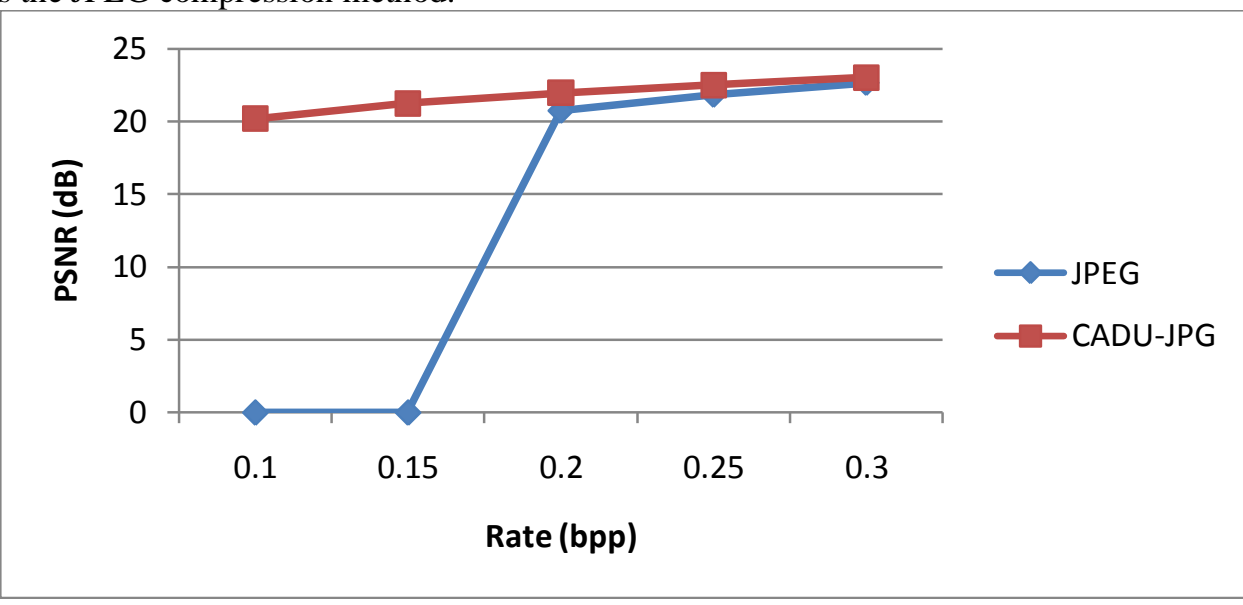

Fig. 5 - Comparison of Techniques With Respect to PSNR with Various Bit Rates (Bike) 
As can be seen in fig 5, the horizontal axis represents bit rate while the vertical axis represents PSNR values. The results reveal that when bit rates are low the compression performance is higher. The proposed approach outperforms the JPEG compression method.

\section{Conclusion}

In this paper, we proposed a new standard for down-sampled image coding which is far better than JPEG 2000 standard in terms visual quality and PSNR. The experiments are made with bit rates between low to medium. A prototype application is built using MATLAB language which proved to be efficient. Its success is attributed to the up conversion process being used by pre-filtering process in the proposed method. The experiments revealed that low bit rates can produce higher image quality. Moreover the proposed approach works faster besides reducing workload and consuming as less energy is possible. The prototype application is very useful in visual communication applications.

\section{References}

[1] E. Cands, "Compressive sampling," in Proc. Int. Congr. Mathematics,Madrid, Spain, 2006, pp. 1433-1452.

[2] X.Wu,K.U. Barthel, and W. Zhang, "Piecewise 2-D autoregression forpredictive image coding," in Proc. IEEE Int. Conf. Image Processing,Chicago, IL, Oct. 1998, vol. 3, pp. 901-904.

[3] X. Li and M. T. Orchard, "Edge-direted prediction for lossless compressionof natural images," IEEE Trans. Image Process., vol. 10, no.6, pp. 813-817, Jun. 2001.

[4] W. Lin and D. Li, “Adaptive downsampling to improve image compressionat low bit rates," IEEE Trans. Image Process., vol. 15, no. 9,pp. 2513-2521, Sep. 2006.

[5] L. Gan, C. Tu, J. Liang, T. D. Tran, and K.-K. Ma, "Undersampledboundary pre-/post-filters for low bit-rate dct-based coders," IEEETrans. Image Process., vol. 16, no. 2, pp. 428-441, Feb. 2007.

[6] X. Zhang, X. Wu, and F. Wu, "Image coding on quincunx lattice withadaptive lifting and interpolation," in Proc. IEEE Data CompressionConf., Mar. 2007, pp. 193-202.

[7] D. Santa-Cruz, R. Grosbois, and T. Ebrahimi, "Jpeg 2000 performanceevaluation and assessment," Signal Process.: Image Commun., vol. 1,no. 17, pp. 113-130, 2002.

[8] A. M. Bruckstein, M. Elad, and R. Kimmel, "Down-scaling for bettertransform compression," IEEE Trans. Image Process., vol. 12, no. 9,pp. 1132-1144, Sep. 2003.

[9] Y. Tsaig, M. Elad, and P. Milanfar, "Variable projection for near-optimalfiltering in low bit-rate block coders," IEEE Trans. Circuits Syst.Video Technol., vol. 15, no. 1, pp. 154-160, Jan. 2005.

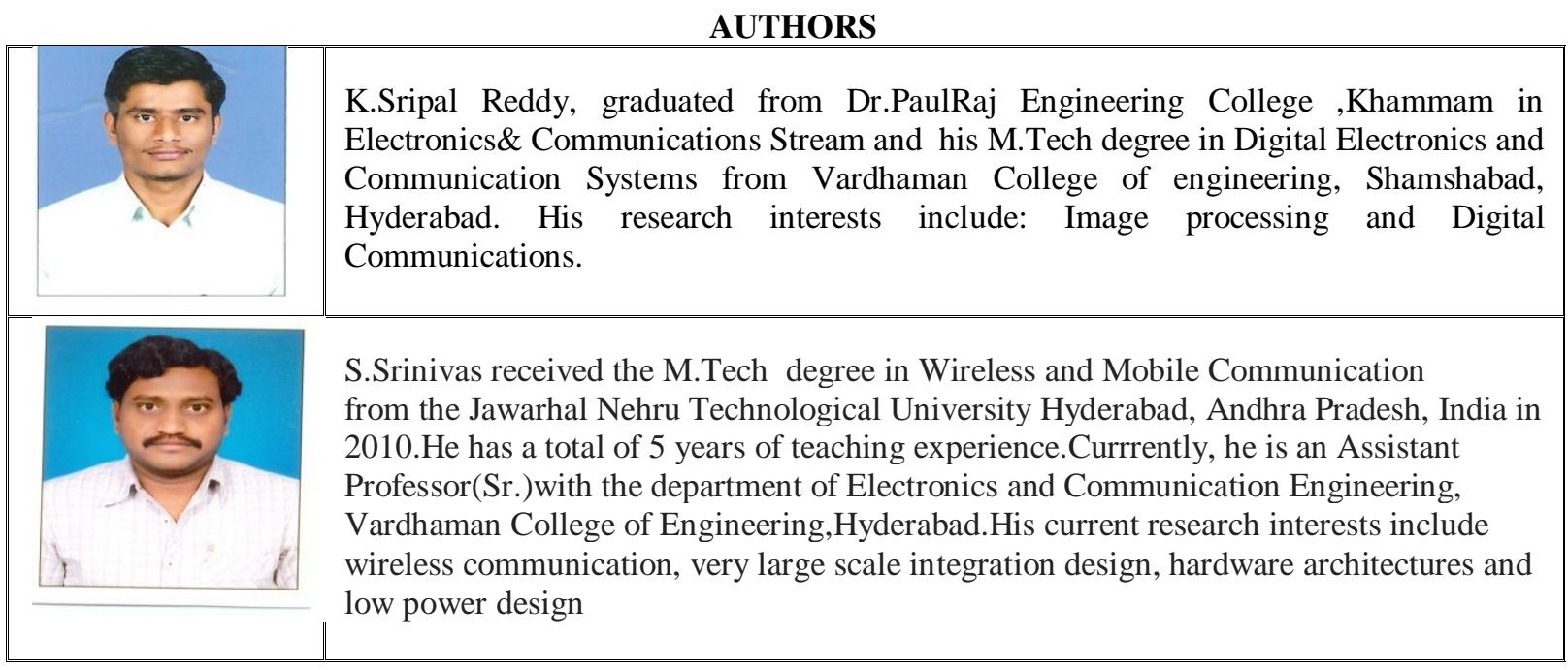

